This is the author's final manuscript of the presented paper. The publisher-formatted version may be available through the publisher's web site or your institution's library

\title{
Wireless slips and falls prediction system
}

Devon Krenzel, Steve Warren, Kejia Li, Bala Natarajan, and Gurdip Singh

\section{How to cite this paper}

If you make reference to this version of the manuscript, use the following information:

Krenzel, D., Warren, S., Li, K., Natarajan, B., \& Singh, G. (2012). Wireless slips and falls prediction system. Retrieved from http://krex.ksu.edu

\section{Published version information}

Citation: Krenzel, D., Warren, S., Li, K., Natarajan, B., \& Singh, G. (2012). Wireless slips and falls prediction system. Engineering in Medicine and Biology Society (EMBC), Annual International Conference of the IEEE, 2012, 4042-4045.

doi: $10.1109 /$ EMBC.2012.6345853

Copyright: (C2012 IEEE

Digital Object Identifier (DOI): doi: 10.1109/EMBC.2012.6345853

\section{Publisher's Link:}

http://ieeexplore.ieee.org/xpl/mostRecentlssue.jsp?punumber=6320834

This item was retrieved from the K-State Research Exchange (K-REx), the institutional repository of Kansas State University. K-REx is available at http://krex.ksu.edu 


\title{
Wireless Slips and Falls Prediction System
}

\author{
Devon Krenzel ${ }^{1}$, Steve Warren ${ }^{1}$, Kejia $\mathrm{Li}^{1}$, Bala Natarajan ${ }^{1}$, and Gurdip Singh $^{2}$ \\ ${ }^{1}$ Department of Electrical \& Computer Engineering, Kansas State University, Manhattan, KS, USA \\ ${ }^{2}$ Department of Computing \& Information Sciences, Kansas State University, Manhattan, KS, USA
}

\begin{abstract}
Accidental slips and falls due to decreased strength and stability are a concern for the elderly. A method to detect and ideally predict these falls can reduce their occurrence and allow these individuals to regain a degree of independence. This paper presents the design and assessment of a wireless, wearable device that continuously samples accelerometer and gyroscope data with a goal to detect and predict falls. Lyapunov-based analyses of these time series data indicate that wearer instability can be detected and predicted in real time, implying the ability to predict impending incidents.
\end{abstract}

Keywords-Accelerometer, gyroscope, wearable devices, ZigBee wireless, Lyapunov exponents, Android smart phone

\section{INTRODUCTION}

Injuries from falls are a concern for the elderly, as approximately one-third of people over the age of 70 fall at least once in a given year, and one-fourth of those falls result in injury [1]. Such injuries can lead to worse conditions if a person is not treated immediately, increasing the chance of fatality within six months after a fall [2]. For this reason, the ability to detect and predict falls is desirable. Research suggests that elderly individuals with gait variability and inconsistencies in their steps fall more often than others, so quantification of an elderly person's balance and walking stability can likely predict their propensity to fall [3]. Fall-prone, elderly patients often decrease walking speed, vary stride time, and lose stability in the hip regions, leading to significantly higher variability in gait patterns [4]. Early work with falls assessment and prediction approaches that utilize Lyapunov methods, e.g., as described in [4], show promise to predict falls up to several hours in advance.

This paper describes a slips-and-falls detection and prediction device designed with Lyapunov methods in mind. The wireless sensor is light weight, compact, and low power so that it can be comfortably worn all day on the patient's hip while it acquires accelerometer and gyroscope data that will be used to assess patient stability via Lyapunov approaches [4-9]. The largest Lyapunov exponents (LLEs) can indicate system stability. By noting the trajectory of the LLEs calculated from patient data, this system will predict if patient gait/balance will become chaotic and unstable, indicating that they may be at risk for a fall.

\section{METHODOLOGY}

\section{A. Device Hardware/Software}

The slips and falls detector/predictor prototype (see Fig. 1) employs a module for wireless communication and processing, an accelerometer and gyroscope for dynamic movement detection, local storage/processing capabilities, and a mini-USB port to recharge the battery and program the processor. If a person has fallen or is at risk to fall, wireless communication is needed to let outside parties know that attention is required. Therefore, a Jennic 5148 ZigBee wireless module (802.15.4 standard at $2.4 \mathrm{GHz}$ ) has been implemented on the sensor board to communicate patient data to an external host, such as a ZigBee-enabled Android smart phone. The patient could then access this information through an Android app. The ZigBee wireless protocol was chosen for its low power consumption, sensible communication range, and adequate throughput. A CMA3000 MEMS 3-axis digital accelerometer and a CMR3000 3-axis digital gyroscope were chosen for their low power consumption, high precision, and high sampling rate. Each of the six sensor axes is sampled at $100 \mathrm{~Hz}$, and data are provided to the Jennic module through a serial port interface. The device can store up to 22 hours of accelerometer/gyroscope data using on-board flash memory. An ABS plastic case for the sensor board is shown in Fig. 2. All sensor data can be received by a PC USB port (either in cabled mode or through a wireless ZigBee dongle) and analyzed in MATLAB. These dynamic-motion plots are utilized to explain concepts in this paper.

Note that while the unit is designed to transmit sensor data and calculations directly to the user's ZigBee-inherent Android smart phone or tablet, market availability of such devices is limited. However, a ZigBee-enabled tablet has been purchased that is compatible with the wireless functionality of the Jennic ZigBee Pro module. An interim solution would be to create a ZigBee receiving dongle that utilizes Android 3.1's ability to support USB host mode, allowing these wireless data to be accepted through the phone's USB port. 


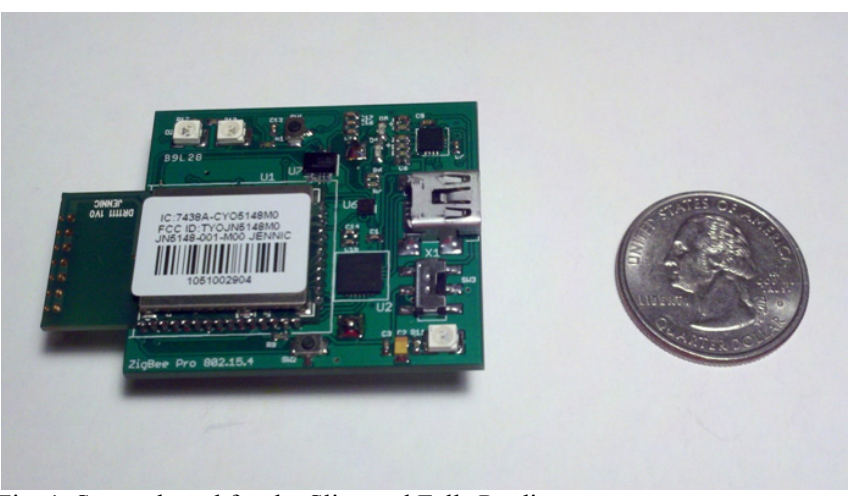

Fig. 1. Sensor board for the Slips and Falls Predictor prototype.

Software decisions can be made to maximize the speed and battery life of the sensor device. For example, since sending data across the ZigBee link consumes the majority of the power on the board, data transmissions to the receiver platform can be limited to (a) the Lyapunov coefficients calculated from the raw sensor data, (b) the slips and falls indicators acquired from those coefficients, and/or (c) low battery level indicators. By limiting data across the link, the battery life can increase from approximately 20 hours (for continuous transmission) to several days on a single charge.

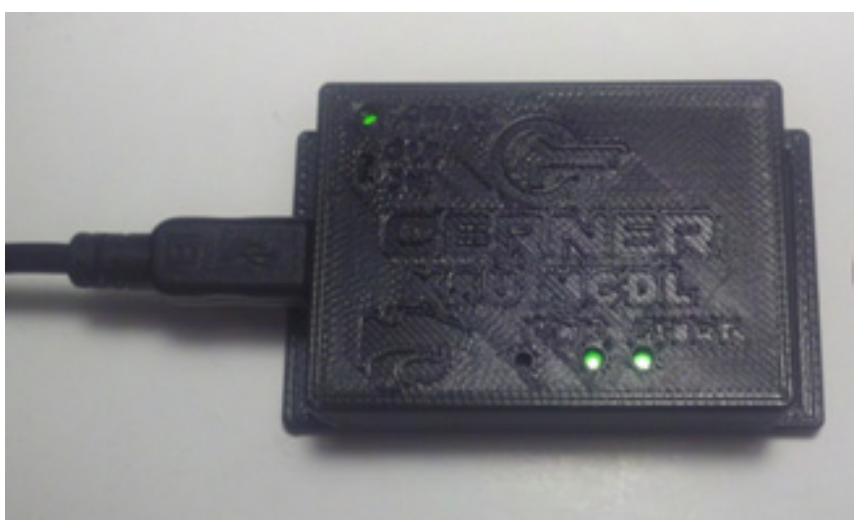

Fig. 2. ABS plastic case for the Slips and Falls Predictor, with a USB cable connected to the board.

\section{B. Lyapunov Algorithm}

To predict if a patient is at risk to fall in the near future, a quick and accurate algorithm needs to be implemented on the device processor. One proposed approach is to execute an algorithm consistent with the family of Lyapunov codes: methods which evaluate the perceived dynamic stability of a system based on current and past time series data, such as the accelerometer and gyroscope data acquired with the Slips and Falls Predictor. Lyapunov exponents measure "exponential rates of average divergence or convergence of nearby trajectories as a system evolves in time" [5]. The hypothesis for this work is that these Lyapunov coefficients will track the instability of a user and allow the system to extrapolate a user's propensity for a fall based on current and past data. This behavior can be modeled by the expression

$$
d(t)=C e^{\lambda t}
$$

where $d(t)$ represents the average divergence at time $t, C$ is the initial separate normalization constant, and $\lambda$ is the spectrum of Lyapunov exponents [6]. Calculating the entire spectrum of exponents allows one to realize the complete stability of the system. However, this is computationally expensive. The calculation can be greatly simplified by realizing that "two randomly selected initial trajectories should diverge, on average, at the rate determined by the largest Lyapunov exponent," or LLE [7]. Calculating only the LLE is much less computationally intensive and can accurately evaluate the stability of a system - in this case, patient gait. A focus on this exponent implies that if the trajectory deviates from the expected trajectory, the system may be chaotic and unstable, indicating that the patient may fall in the near future. In other words, the value of an exponent indicates how quickly the trajectory departs from the nominal trajectory [8]. Therefore, if the algorithm yields a higher, positive value of the LLE, the trajectory will be assumed to exponentially deviate even more quickly, indicating a more unstable system. If the LLE is equal to (or very close to) zero, the system is stable, and if it is negative, the system is stationary [9]. When the average expansion rate of the trajectory demonstrates a strong linear increase in the given time series, the slope will be used to calculate the LLE. Factors such as noise, undersized time series, and small embedding dimensions can result in an absent linear region, resulting in a miscalculation of an LLE [9]. Many Lyapunov methods can be utilized to analyze the stability of patient dynamics, but Rosenstein's method to calculate LLEs is most appropriate for this situation. This is because Rosenstein's approach is more reliable with small data sets, less computationally expensive, and less sensitive to noise compared to other similar algorithms [6].

The accuracy and speed of Rosenstein's method to calculate LLEs depend on several factors, such as the size of the embedding dimension, the embedding lag, and the length of the time series. Each of these factors can be calculated or estimated, depending on the sample data. Since the LLE could potentially be calculated up to several hundred times per second for this application, the length of the time series data set inserted into the algorithm is important. A greater number of time series samples used for each LLE calculation yields greater accuracy, but at the cost of increased computation time. However, while smaller time series lead to faster computations, the resulting LLEs may be inaccurate.

Fig. 3 below shows the calculated LLEs versus the number of accelerometer samples inserted into the Lyapunov algorithm for a person walking both stably and unstably. The number of samples ranges from $50(0.5$ seconds of walking) to 10,000 (100 seconds of walking). Clearly, for a small time series, the LLE is inaccurate and inconsistent with LLEs obtained using larger sample sizes. As greater numbers of samples are introduced into the 
algorithm, the LLEs begin to stabilize and converge to a consistent value. Given the information in Fig. 3, it is unnecessary to introduce more than 5,000 samples into the Lyapunov algorithm, as the algorithm will continue to yield approximately the same LLEs [5]. Also, note that the unstable, more chaotic walking pattern did indeed yield higher LLEs than walking stably.

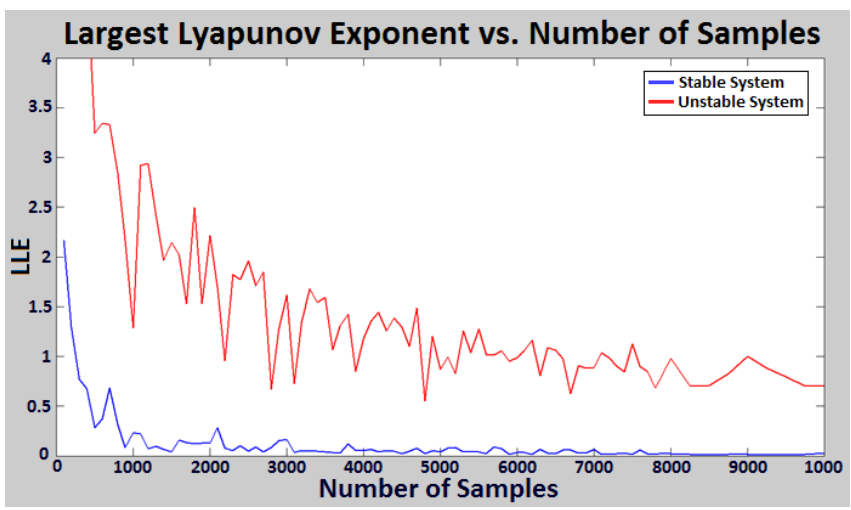

Fig. 3. Calculated LLEs versus the length of the time series data for both stable (blue) and unstable (red) walking.

\section{RESULTS AND DISCUSSION}

\section{A. Instability Detection}

Accurately distinguishing between stable and unstable dynamic movements is important in this application because false negatives (non-perceived instabilities) could lead to falls and injuries. Therefore, accurate calculations of LLEs are crucial. Fig. 4 displays short time series for all three axes of both accelerometer and gyroscope data for a patient walking consistently and stably, while Fig. 5 displays similar data for a patient walking unstably and chaotically. Note how a consistent pattern emerges from the sensor data in both cases. Therefore, when applying Rosenstein's method for the Lyapunov algorithm, the calculated LLEs tend to be very small because very little deviation and few inconsistencies exist in the time series data. However, inconsistent data, as shown in Fig. 5, yield higher LLEs because of deviations from the nominal trajectory. This phenomenon is summarized in Fig. 6, which shows the LLEs of each axis under both stable and unstable conditions. In early studies, the LLEs for an unstable system can be approximately fifteen times greater than those of a stable system. To obtain these Fig. 6 values, 5,000 data samples were entered into the Lyapunov algorithm, along with an embedding dimension of five and an embedding lag of one.

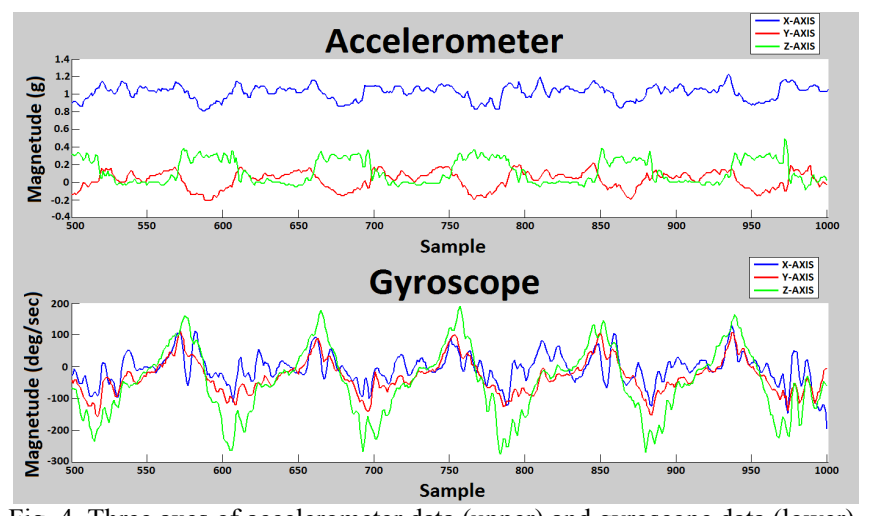

Fig. 4. Three axes of accelerometer data (upper) and gyroscope data (lower) from a patient walking stably.

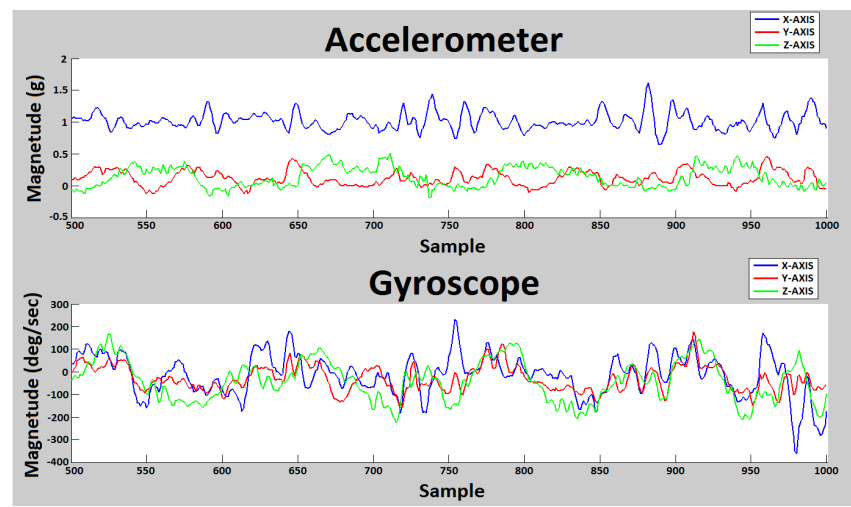

Fig. 5. Three axes of accelerometer data (upper) and gyroscope data (lower) from a patient walking unstably.

\begin{tabular}{|c|c|c|c|c|}
\hline \multicolumn{4}{|c|}{ Largest Lyapunov Exponents for Sensor Time Series } \\
\hline & \multicolumn{2}{|c|}{ Stable Walking } & \multicolumn{2}{c|}{ Unstable Walking } \\
\hline & Accelerometer & Gyroscope & Accelerometer & Gyroscope \\
\hline X-Axis & 0.0597 & 0.0378 & 1.013 & 0.868 \\
\hline Y-Axis & 0.0951 & 0.0761 & 1.202 & 1.165 \\
\hline Z-Axis & 0.0668 & 0.0804 & 0.991 & 0.976 \\
\hline
\end{tabular}

Fig. 6. Calculated LLEs for both stable and unstable walking.

\section{B. Slip and Fall Detection}

Not only should the device predict possible future falls through instabilities, but it should also detect whether the subject experiences a sudden, accidental slip or fall. Therefore, it is important to distinguish between falls and harmless movements. The two scenarios can be distinguished by several dynamic features in the sensor data. Fig. 7 displays accelerometer and gyroscope data for a person sitting in a chair, while Fig. 8 displays these data for a person falling to the ground. The two situations can be distinguished from one another by the rate of axis transitions and the amplitudes of the curves. When the subject is sitting down, the transitions of the accelerometer and gyroscope data are smoother and have smaller magnitudes. When the subject is falling to the ground, the data have jagged transitions and exhibit large spikes in amplitude. Furthermore, the falling data are characterized by a moment 
of "weightlessness" (where all three axes of the accelerometer are close to zero), a large rotational value for the Z-axis of the gyroscope (yaw), and a large spike in the amplitude of the data from both sensors, signifying the impact with the ground [10].

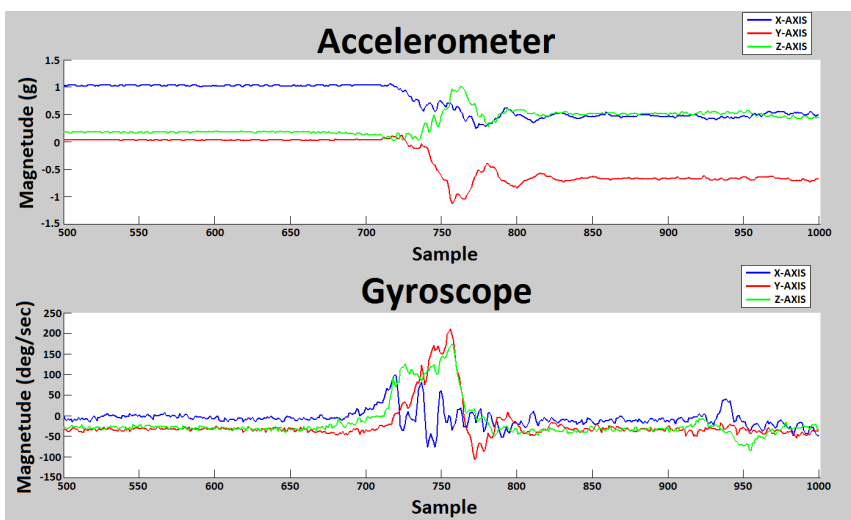

Fig. 7. Three axes of accelerometer and gyroscope data for a person sitting in a chair.

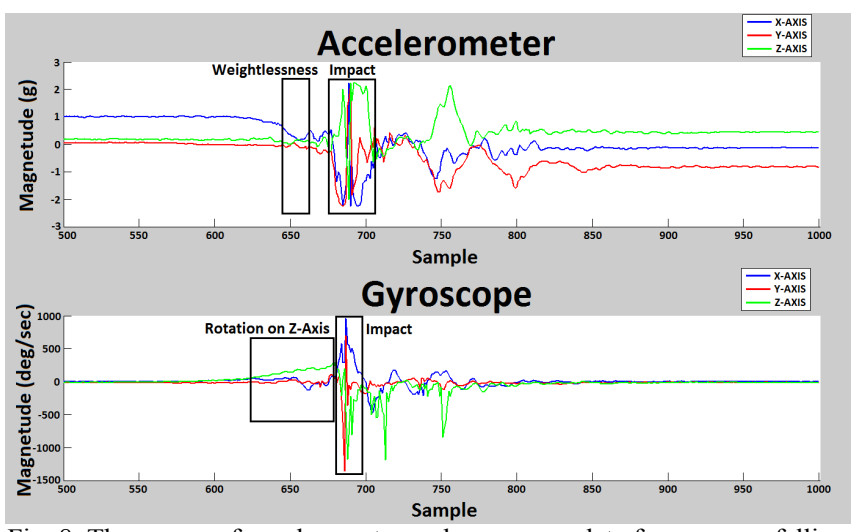

Fig. 8. Three axes of accelerometer and gyroscope data for a person falling backwards to the ground.

\section{CONCLUSION}

Accurately analyzing and applying largest Lyapunov exponents (LLEs) calculated from accelerometer and gyroscope time series data has the potential to predict falls due to instability and therefore avoid possible serious injuries. This paper introduced and evaluated a prototype slips-and-falls detection and prediction device that can be comfortably worn while accelerometer and gyroscope sensor data are collected. Not only could this device predict potential falls, but it also offers the potential to distinguish between falls and normal dynamic movements. By utilizing Rosenstein's method to calculate LLEs, the stability of a system can be accurately and quickly assessed with relatively small data sets. Upon careful implementation of this algorithm, falls will ideally be predicted several hours in advance. Furthermore, falls can also be detected by analyzing small data sets from the accelerometer and gyroscope. By detecting signs of weightlessness, swift axial rotation, and large sensor magnitudes from impact, falls can accurately be detected without false alarms. With the type of system presented here, these vital data could be sent to a patient's or caretaker's Android cell phone to let them know that attention is required. Precisely detecting falls and potential falls may allow senior citizens to regain a level of reassurance and independence.

\section{REFERENCES}

[1] J. Liu, et al. "Local Dynamic Stability Assessment of Motion Impaired Elderly Using Electronic Textile Pants," IEEE Transactions on Automation Science and Engineering, vol. 5, no. 4, pp. 696-702, Oct. 2008.

[2] J.T. Perry, et al. "Survey and Evaluation of Real-Time Fall Detection Approaches," High-Capacity Optical Networks and Enabling Technologies (HONET), pp. 158, December 2009.

[3] R. M. Guimaraes, B. Isaacs. "Characteristics of the Gait in Old People Who Fall," Int. Rehab. Med., vol. 2, pp. 177-180, 1980.

[4] C.L. Lamoth, et al. "Gait Stability and Variability Measures Show Effects of Impaired Cognition and Dual Tasking in Frail People," Journal of NeuroEngineering and Rehabilitation, 2011 8:2.

[5] T.M. TenBroek, et al. "Sensitivity of Lyapunov Exponent Estimation for Human Gait," Department of Kinesiology. Amherst: University of Massachusetts Amherst, 2006.

[6] M.T. Rosenstein, et al. "A Practical Method for Calculating Largest Lyapunov Exponents from Small Data Sets," Physica D: Nonlinear Phenomena, vol. 65, pp. 117-134, Nov. 1992.

[7] S.A. England, K.P. Granata. "The Influence of Gait Speed on Local Dynamic Stability of Walking," Gait Posture, vol. 25, no. 2, pp. 172178, Feb. 2007.

[8] E.A. Best. "Stability Assessment of Nonlinear Systems Using the Lyapunov Exponent," College of Engineering and Technology. Athens: University of Ohio, June 2003.

[9] S. Ohgi, et al. "Time Series Analysis of Spontaneous UpperExtremity Movements of Premature Infants with Brain Injuries," Phys Ther, vol. 88, pp. 1022-1033, July 2008.

[10] G. Brown. "An Accelerometer Based Fall Detector: Development, Experimentation, and Analysis," EECS/SUPERB, July 2005. 